\title{
Medium-term Influence of the Coronavirus Disease 2019 Pandemic on Patients with Diabetes: A Single-center Cross-sectional Study
}

\author{
Yumiko Maruo, Yoko Irie, Yoshinari Obata, Kana Takayama, Hiroki Yamaguchi, \\ Motohiro Kosugi, Yoji Hazama and Tetsuyuki Yasuda
}

\begin{abstract}
:
Objective This study evaluated the lifestyle changes in patients with diabetes and their independent associations with glycemic and body weight control. In addition, the correlation between changes in mental health and lifestyles was evaluated.

Methods This single-center cross-sectional study included 340 patients with diabetes who periodically visited our department. Changes in dietary habits, activities of daily living, and mental health before and during approximately six months after the onset of the coronavirus disease 2019 (COVID-19) pandemic were evaluated using a questionnaire, including the International Physical Activity Questionnaire-Short Form.

Results Approximately 20\%,30\%, and over 50\% of patients had worsened dietary habits, decreased activities of daily living, and deteriorated mental health, respectively. A multiple regression analysis showed that irregular meal timing was significantly associated with change in $\operatorname{HbAlc}(\beta=0.328, \mathrm{p}=0.001)$, and decreased walking time was significantly associated with changes in body weight $(\beta=-0.245, p=0.025)$. The change in fear and anxiety was positively associated with changes in meal timing regularity $(r=0.129, p=0.019)$ and carbohydrate consumption $(\mathrm{r}=0.127, \mathrm{p}=0.021)$. Subsequently, the change in depressed mood was positively associated with changes in carbohydrate $(\mathrm{r}=0.142, \mathrm{p}=0.010)$ and alcohol $(\mathrm{r}=0.161, \mathrm{p}=0.037)$ consumption, and the change in psychological stress was positively associated with changes in carbohydrates $(r=0.183, p=0.001)$ and snack ( $\mathrm{r}=0.151, \mathrm{p}=0.008)$ consumption as well as sedentary time $(\mathrm{r}=0.158, \mathrm{p}=0.004)$.

Conclusion The COVID-19 pandemic has had a considerable medium-term impact on the lifestyle and mental health of patients with diabetes. Lifestyle changes were associated with glycemic and body weight control, and mental health changes were associated with lifestyle changes. These findings may provide important information on diabetes care during the pandemic.
\end{abstract}

Key words: COVID-19, diabetes, lifestyle, mental health

(Intern Med 61: 303-311, 2022)

(DOI: 10.2169/internalmedicine.8010-21)

\section{Introduction}

The novel coronavirus disease 2019 (COVID-19) has caused a global health emergency; governments of pandemic-hit countries have adopted different measures to prevent the spread of the disease and the collapse of the healthcare system. Depending on the rate of transmission and the robustness of the medical system, these measures range from requests to promote social distancing to forced lockdowns of cities, with penalties for violations (1).

In Japan, the government declared a state of emergency over the COVID-19 outbreak, requesting that residents refrain from nonessential outings and restricting certain business operations without penalties for violations in seven prefectures, including Osaka, on April 7, 2020. This declaration was expanded nationwide on April 16, 2020, and phased out due to a reduction in the first wave of transmission on May 
14, 2020 (2). This situation temporarily affected Japanese people's lifestyle and mental health, including their dietary habits and activities of daily living $(3,4)$. However, the second and third waves of infection have since begun, and as of December 31, 2020, Japan had recorded 233,785 confirmed COVID-19 cases and 3,459 deaths (2).

For patients with diabetes, dietary habits, activities of daily living, and mental health are particularly important in disease management, and any disruptive change is likely to adversely affect patients' glycemic and body weight control $(5,6)$. Indeed, recent studies have evaluated the impact of the COVID-19 pandemic on the changes in lifestyles and/ or mental health and their impact on glycemic and body weight control (7-14). However, these reports evaluated the early phase, approximately two months after the beginning of the COVID-19 pandemic. The medium-term impact of the COVID-19 pandemic on changes in lifestyles and mental health and their independent impact on glycemic and body weight control in patients with diabetes thus remain unclear. Furthermore, the correlation between changes in mental health and lifestyle also remains unclear.

This single-center, cross-sectional study evaluated the lifestyle and mental health changes and their independent association with glycemic and body weight control in patients with diabetes during the medium-term period after the beginning of the COVID-19 pandemic. In addition, the correlation between mental health and lifestyle changes was also evaluated.

\section{Materials and Methods}

\section{Study design and participants}

This single-center, cross-sectional study was conducted at the Department of Diabetes, Metabolism, and Endocrinology of the Osaka Police Hospital in Japan. Patients with diabetes over 20 years old who periodically visited our department and provided their written consent to participate in the study were included. Individuals with malignant tumors, mental illnesses, or dementia and those with serious illnesses or conditions that significantly affected their daily lives were excluded or deemed ineligible for participation by an attending physician. Demographic data and anthropometric measurements were obtained from medical records at the time the questionnaire survey was conducted, from September 1 to October 30, 2020.

\section{Questionnaire}

The questionnaire was developed by our department and consisted of three sections. The first section contained questions on changes in dietary habits (regularity of meal timings, amount of total diet, and consumption of carbohydrates, snacks, fruits, and alcohol) during the pandemic (from April 2020 to the time the questionnaire was completed) compared to before the pandemic. The responses to the question on meal timing regularity included "became regular," "no change," or "became irregular." The responses to the question on dietary habits included "decreased," "no change," or "increased."

The second section contained questions regarding activities of daily living, including physical activity and sedentary time before and during the pandemic, which were measured using the International Physical Activity Questionnaire-Short Form (IPAQ-SF) (15). This questionnaire comprises seven questions assessing the total time (in minutes) spent on vigorous-/moderate-intensity activities and walking per week and being sedentary per day. The responses regarding the vigorous-/moderate-intensity activities and walking were converted to metabolic equivalent task minutes per week (MET.min/week), using the IPAQ scoring protocol. The total weekly physical activity level was estimated by adding the scores for each activity. The last section contained questions regarding changes in mental health, including fear and anxiety, depressed mood, and psychological stress during the pandemic compared to before the pandemic. The three-item responses included "decreased," "no change," or "increased." The respondents completed the questionnaire at regular visits during the pandemic (September 1 to October 30, 2020).

\section{Changes in HbA1c, body weight, and hypoglycemic agents}

The change in HbAlc was calculated as the difference in this indicator at the time of questionnaire completion during the pandemic from that at the regular visits within nearly two months before the state of emergency over the COVID19 outbreak (April 7, 2020). The same calculation applied to the change in body weight. The change in hypoglycemic agents was evaluated as "strengthened," "unchanged/modified," or "attenuated" during the pandemic (from April 7, 2020 , to the time of questionnaire completion).

\section{Ethics statement}

The protocol was approved by the Osaka Police Hospital Clinical Research Review Committee in compliance with the Declaration of Helsinki and the current legal regulations in Japan. Written informed consent was obtained from all participants before participation in the study.

\section{Statistical analyses}

Statistical analyses were performed using the IBM SPSS Statistics for Windows, version 21.0, software program (IBM, Armonk, USA). Continuous variables are presented as means and standard deviations, while categorical variables are presented as valid percentages. The changes in dietary habits, mental health, and hypoglycemic agents were coded as follows: regularity of meal timing was $-1=$ became regular, $0=$ no change, and $1=$ became irregular. Other dietary habits were coded as $-1=$ decreased, $0=$ no change, and $1=$ increased. Mental health was coded as $-1=$ decreased, $0=$ no change, and $1=$ increased. Hypoglycemic agents were as follows: $-1=$ strengthened, $0=$ unchanged/modified, and $1=$ attenuated. Differences in physical activity and sedentary time 
before and during the pandemic were analyzed using Wilcoxon's signed-rank test. A Pearson's correlation analysis and multiple regression analysis were used to evaluate if and how lifestyle changes were associated with those in HbAlc and body weight. A Pearson's correlation analysis was also used to examine the correlation between mental health and lifestyle changes. Statistical significance was set at $\mathrm{p}<0.05$.

Table 1. Demographic Characteristics and Anthropometric Measurements.

\begin{tabular}{lc}
\hline \multicolumn{1}{c}{ Variables } & Value \\
\hline N (Male/female) & $340(226 / 114)$ \\
Age (years) & $67.2 \pm 11.2$ \\
Type of diabetes (type 2/type 1/others) & $320 / 15 / 5$ \\
Duration of diabetes (years) & $16.3 \pm 11.6$ \\
Body weight (kg) & $68.8 \pm 14.5$ \\
BMI (kg/m $\left.{ }^{2}\right)$ & $25.5 \pm 4.3$ \\
Alcohol (yes/no) (\%) & $40.0 / 60.0$ \\
Smoking (yes/no) $(\%)$ & $17.1 / 82.9$ \\
Established cardiovascular disease (yes/no) (\%) & $32.9 / 67.1$ \\
Employment (yes/no) (\%) & $48.4 / 51.6$ \\
HbA1c (\%) & $7.0 \pm 0.8$ \\
Systolic blood pressure (mmHg) & $131.6 \pm 15.3$ \\
Diastolic blood pressure (mmHg) & $77.4 \pm 11.6$ \\
eGFR (mL/min/1.73m $\left.{ }^{2}\right)$ & $67.7 \pm 20.7$ \\
Diabetes treatment & \\
Diet only (\%) & 2.1 \\
Oral hypoglycemic agents (\%) & 90.6 \\
GLP-1RAs (\%) & 25.0 \\
Insulin (\%) & 25.0 \\
\hline
\end{tabular}

Data are presented as means \pm standard deviation (SD) or proportion. BMI: body mass index, eGFR: estimated glomerular filtration ratio, GLP-1RAs: glucagon-like peptide-1 receptor agonists, HbAlc: glycated hemoglobin Alc

\section{Results}

\section{Demographic characteristics and anthropometric measurements}

A total of 360 patients completed the questionnaire, with HbA1c and body weight changes evaluated in 340. Overall, $226(66.5 \%)$ were men, and the majority $(94.1 \%)$ had type 2 diabetes. Their mean age was $67.2 \pm 11.2$ years old; most patients $(63.8 \%)$ were between 60 and 79 years old, followed by those between 40 and 59 years old (22.6\%). Patients' mean body mass index (BMI) was $25.5 \pm 4.3 \mathrm{~kg} / \mathrm{m}^{2}$. The mean duration of diabetes and HbA1c levels was $16.3 \pm 11.6$ years and $7.0 \% \pm 0.8 \%$, respectively. Established cardiovascular diseases were present in $32.9 \%$ of patients. The summarized demographic data and anthropometric measurements of all patients are presented in Table 1.

\section{Changes in dietary habits during the pandemic}

The changes in dietary habits before and during the pandemic are shown in Fig. 1. Changes in the regularity of meal timing showed that $15.2 \%$ of patients reported regular habits, whereas $7.5 \%$ reported irregular meal timings. Total diet decreased in $11.8 \%$ of patients but increased in $13.0 \%$ during the pandemic. The proportions of patients who reported reduced carbohydrate, snack, and fruit consumption were $6.0 \%, 9.1 \%$, and $12.9 \%$, while $22.2 \%, 15.3 \%$, and $21.8 \%$ reported increased consumption, respectively. Alcohol consumption decreased and increased in $24.9 \%$ and $10.1 \%$ of patients, respectively.

\section{Activities of daily living before and during the pan- demic}

Table 2 presents the results of the IPAQ-SF, which evalu-

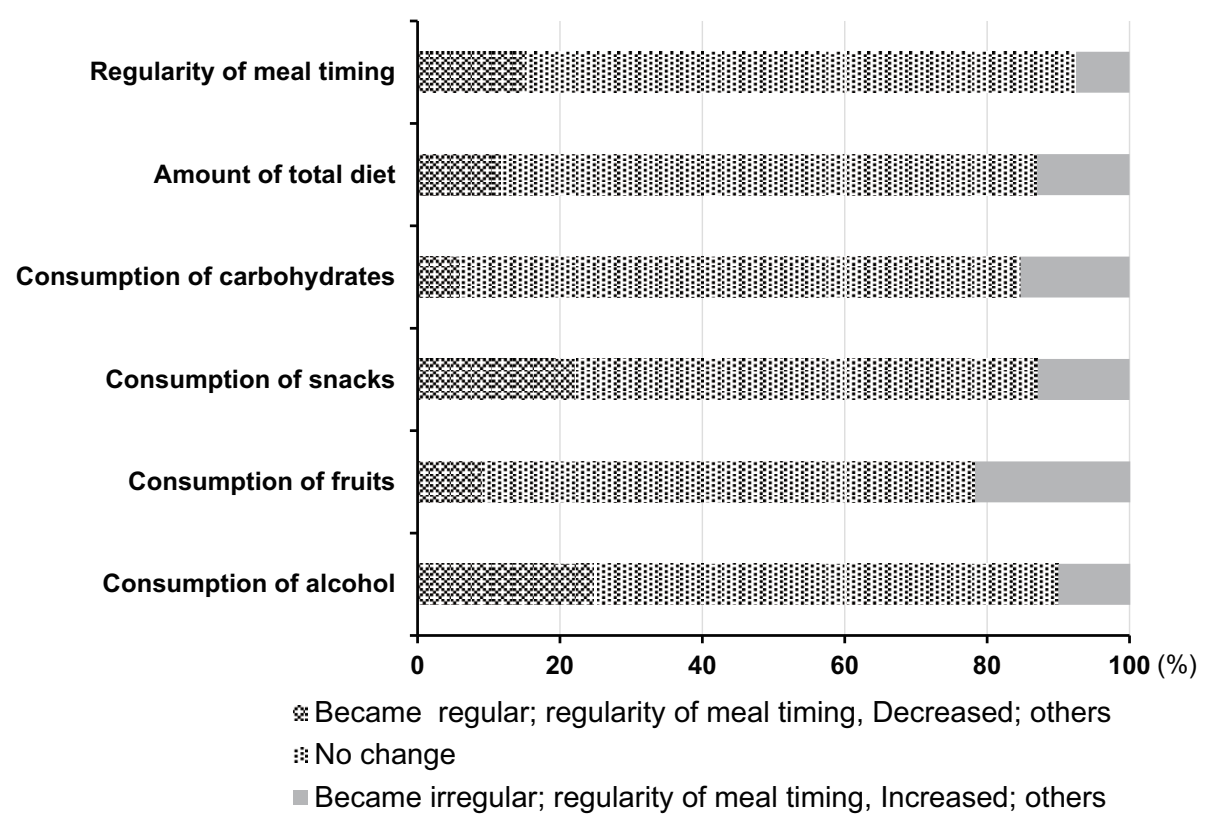

Figure 1. The changes in dietary habits during the pandemic. 
Table 2. Activities of Daily Living before and during the Pandemic.

\begin{tabular}{lccc}
\hline & Before the pandemic & During the pandemic & p value \\
\hline Vigorous intensity (METs.min/week) & $1,220.1 \pm 2,582.9$ & $759.3 \pm 2,050.6$ & $<0.001$ \\
Moderate intensity (METs.min/week) & $466.9 \pm 1,468.8$ & $297.8 \pm 1,150.4$ & $<0.001$ \\
Walking (METs.min/week) & $959.8 \pm 1,363.9$ & $769.6 \pm 1,405.1$ & $<0.001$ \\
All physical activity (METs.min/week) & $2,592.6 \pm 4,333.5$ & $1,803.5 \pm 3,339.0$ & $<0.001$ \\
Sedentary time (min/day) & $305.1 \pm 218.5$ & $353.6 \pm 230.7$ & $<0.001$ \\
\hline
\end{tabular}

Physical activity and sedentary time were measured using the International Physical Activity QuestionnaireShort Form (IPAQ-SF).

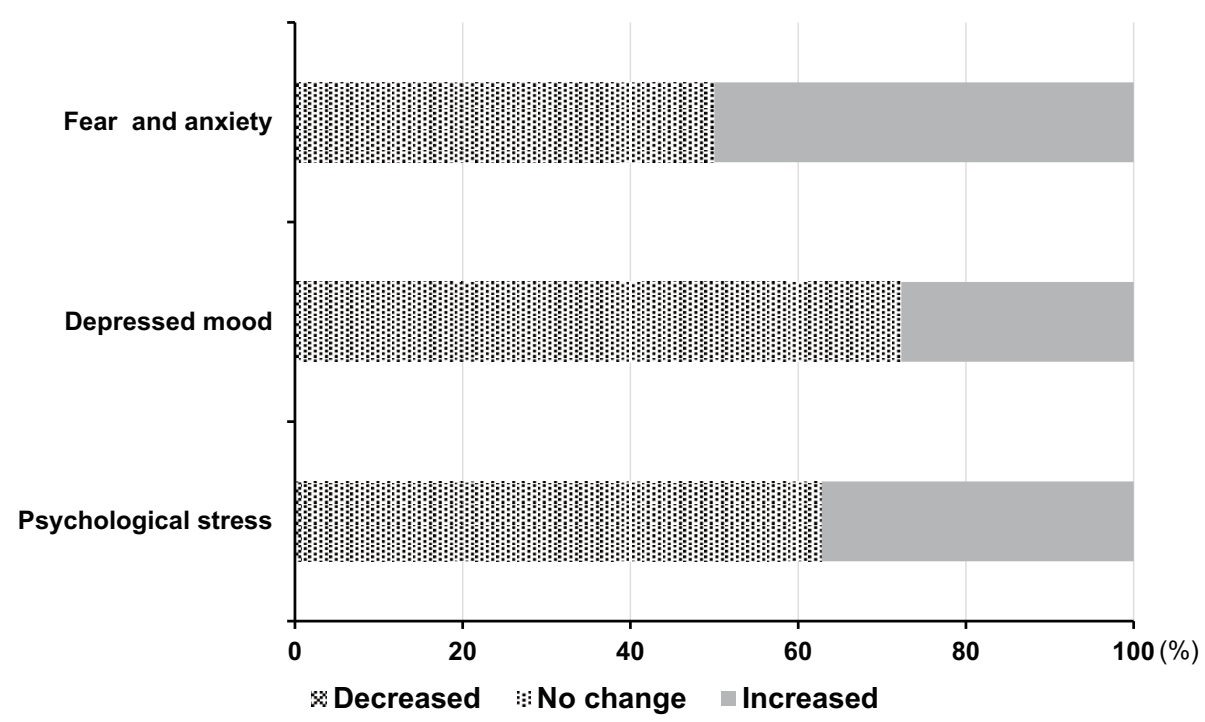

Figure 2. The changes in mental health, including fear and anxiety, depressed mood, and psychological stress during the pandemic.

ated patients' activities of daily living, including physical activity and sedentary time, before and during the pandemic. The proportions of patients with decreased and increased physical activity were $32.6 \%$ and $4.1 \%$, respectively. Vigorous-/moderate-intensity activities and walking during the pandemic were significantly decreased compared to those before the pandemic (vigorous: 759.3 $\pm 2,050.6 \mathrm{MET}$. $\mathrm{min} /$ week vs. 1,220.1 $\pm 2,582.9$ MET.min/week, $\mathrm{p}<0.001$; moderate: $297.8 \pm 1,150.4$ MET.min/week vs. $466.9 \pm 1,468.8$ MET.min/week, p<0.001, walking; 769.6 $\pm 1,405.1$ MET.min/ week vs. 959.8 $\pm 1,363.9$ MET.min/week, $\mathrm{p}<0.001$, respectively). Furthermore, total physical activity during the pandemic was significantly decreased compared to before the pandemic $(1,803.5 \pm 3,339.0$ MET.min/week vs. 2,592.6 \pm 4,333.5 MET.min/week, $\mathrm{p}<0.001)$.

The sedentary time increased and decreased in $33.8 \%$ and $1.5 \%$ of patients, respectively. The sedentary time per day during the pandemic was significantly increased compared to before the pandemic $(353.6 \pm 230.7 \mathrm{~min} /$ day vs. $305.1 \pm$ $218.5 \mathrm{~min} /$ day, $\mathrm{p}<0.001)$.

\section{Changes in mental health during the pandemic}

Regarding changes in mental health, increased fear and anxiety, depressed mood, and psychological stress were re- ported in $50.0 \%, 27.7 \%$, and $37.2 \%$ of patients, respectively during the pandemic (Fig. 2).

\section{Hypoglycemic agent changes during the pandemic}

During the pandemic, the usage of hypoglycemic agents was strengthened, unchanged/modified, and attenuated in $10.0 \%, 82.4 \%$, and $7.6 \%$, respectively.

\section{Association of lifestyle changes and mental health with changes in HbA1c and body weight}

The mean HbA1c value slightly but significantly decreased during the pandemic $(7.09 \% \pm 0.73 \%$ to $6.94 \%$ $\pm 0.73 \%, \mathrm{p}<0.001)$. In contrast, the body weight did not significantly vary during the pandemic compared with before the pandemic $(69.0 \pm 14.4 \mathrm{~kg}$ vs. $68.8 \pm 14.5 \mathrm{~kg}, \mathrm{p}=0.267)$. Tables 3 and 4 describe how lifestyle changes were related to $\mathrm{HbA1c}$ and body weight changes. Irregular meal timing was positively associated, but changes in walking time were negatively associated with changes in HbA1c $(\beta=0.202, p=<$ 0.001 , and $\mathrm{r}=-0.116, \mathrm{p}=0.048$, respectively).

A multiple regression analysis included all items from the questionnaire and patient attributes (age, sex, BMI, duration of diabetes, and changes in hypoglycemic agents) as explanatory variables and the change in the HbAlc as an ob- 
Table 3. The Association of Changes in Lifestyles and Mental Health with the Changes in HbA1c $(\mathbf{n}=340)$.

\begin{tabular}{|c|c|c|c|c|c|c|}
\hline & \multicolumn{2}{|c|}{ Univariate } & \multicolumn{2}{|c|}{$\begin{array}{l}\text { Regression coefficient } \\
\text { in ordinal variables }\end{array}$} & \multicolumn{2}{|c|}{ Multivariate } \\
\hline & $\mathrm{r}$ & $\mathrm{p}$ & $\beta$ & $\mathrm{p}$ & $\beta$ & $\mathrm{p}$ \\
\hline Age (years) & 0.016 & 0.774 & & & -0.039 & 0.714 \\
\hline $\operatorname{Sex}(0=$ Female, $1=$ Male $)$ & -0.067 & 0.224 & & & -0.144 & 0.109 \\
\hline Duration of diabetes (years) & -0.079 & 0.149 & & & 0.091 & 0.350 \\
\hline $\operatorname{BMI}\left(\mathrm{kg} / \mathrm{m}^{2}\right)$ & 0.085 & 0.122 & & & -0.035 & 0.748 \\
\hline Employment ( $0=$ No, $1=$ Yes $)$ & -0.067 & 0.223 & & & & \\
\hline \multicolumn{7}{|l|}{ Change in hypoglycemic agents } \\
\hline Unchanged/modified vs. strengthened & & & 0.014 & 0.801 & 0.019 & 0.842 \\
\hline Attenuated vs. unchanged/modified & & & -0.034 & 0.543 & 0.182 & 0.068 \\
\hline \multicolumn{7}{|l|}{ Changes in dietary habits } \\
\hline \multicolumn{7}{|l|}{ Regularity of meal timing } \\
\hline Unchanged vs. became regular & & & -0.035 & 0.52 & 0.054 & 0.602 \\
\hline Became irregular vs. unchanged & & & 0.202 & $<0.001$ & 0.328 & 0.001 \\
\hline \multicolumn{7}{|l|}{ Amount of total diet } \\
\hline Unchanged vs. decreased & & & 0.052 & 0.349 & -0.104 & 0.323 \\
\hline Increased vs. unchanged & & & 0.107 & 0.056 & 0.053 & 0.656 \\
\hline \multicolumn{7}{|l|}{ Consumption of carbohydrates } \\
\hline Unchanged vs. decreased & & & -0.049 & 0.383 & 0.074 & 0.495 \\
\hline Increased vs. unchanged & & & 0.039 & 0.486 & -0.151 & 0.156 \\
\hline \multicolumn{7}{|l|}{ Consumption of snacks } \\
\hline Unchanged vs. decreased & & & -0.076 & 0.182 & 0.165 & 0.111 \\
\hline Increased vs. unchanged & & & -0.021 & 0.713 & -0.063 & 0.506 \\
\hline \multicolumn{7}{|l|}{ Consumption of fruits } \\
\hline Unchanged vs. decreased & & & -0.103 & 0.067 & -0.155 & 0.122 \\
\hline Increased vs. unchanged & & & 0.023 & 0.685 & -0.101 & 0.294 \\
\hline \multicolumn{7}{|l|}{ Consumption of alcohol } \\
\hline Unchanged vs. decreased & & & -0.041 & 0.611 & -0.052 & 0.614 \\
\hline Increased vs. unchanged & & & 0.058 & 0.469 & -0.020 & 0.836 \\
\hline \multicolumn{7}{|l|}{ Changes in activities of daily living } \\
\hline Vigorous intensive activity & -0.009 & 0.869 & & & -0.029 & 0.825 \\
\hline Moderate intensive activity & 0.011 & 0.854 & & & 0.143 & 0.267 \\
\hline Walking & -0.116 & 0.048 & & & -0.146 & 0.166 \\
\hline Total activity & -0.028 & 0.644 & & & & \\
\hline Sedentary time & 0.077 & 0.173 & & & -0.015 & 0.885 \\
\hline \multicolumn{7}{|l|}{ Changes in mental health } \\
\hline \multicolumn{7}{|l|}{ Fear and anxiety } \\
\hline Unchanged vs. decreased & & & 0.038 & 0.498 & -0.114 & 0.307 \\
\hline Increased vs. unchanged & & & 0.033 & 0.552 & 0.064 & 0.563 \\
\hline \multicolumn{7}{|l|}{ Depressive mood } \\
\hline Unchanged vs. decreased & & & 0.106 & 0.054 & 0.228 & 0.207 \\
\hline Increased vs. unchanged & & & 0.053 & 0.339 & 0.056 & 0.661 \\
\hline \multicolumn{7}{|l|}{ Psychological stress } \\
\hline Unchanged vs. decreased & & & 0.064 & 0.248 & -0.117 & 0.489 \\
\hline Increased vs. unchanged & & & 0.053 & 0.338 & 0.032 & 0.799 \\
\hline
\end{tabular}

HbA1c: glycated hemoglobin A1c, BMI: body mass index

Change in hypoglycemic agents was coded as "strengthened" (-1 point), "unchanged/modified" (0 point), and "attenuated"(1 point).

Each lifestyle change was coded as "decreased/became regular" (-1 point), "unchanged" (0 point), and "increased/became irregular" (1 point)

jective variable. Irregular meal timing was identified as a the BMI $(r=0.187, p=0.001)$, irregular meal timing ( $\beta=0.190$, factor significantly related to the change in $\operatorname{HbA1c}(\beta=\mathrm{p}=0.001)$, changes in total diet $(\mathrm{r}=0.158, \mathrm{p}=0.004)$, carbohy0.328, $\mathrm{p}=0.001$ ) (Table 3). In contrast, some factors, such as drate consumption $(\mathrm{r}=0.120, \mathrm{p}=0.029)$, fear and anxiety $(\mathrm{r}=$ 
0.162, $\mathrm{p}=0.003)$, and psychological stress $(\mathrm{r}=0.122, \mathrm{p}=$ 0.026), were positively associated with body weight change. However, only the change in walking time showed an inverse association with body weight change $(\mathrm{r}=-0.154, \mathrm{p}=$ 0.008).

With all items of the questionnaire and patient attributes (age, sex, BMI, duration of diabetes, and changes in hypoglycemic agents) included as explanatory variables and the change in body weight as an objective variable, we performed a multiple regression analysis. This analysis revealed that only the change in walking time significantly contributed to body weight change $(\beta=-0.245, \mathrm{p}=0.025)$ (Table 4).

\section{Correlation between mental health and lifestyle changes}

The correlations between mental health and lifestyle changes are shown in Table 5. The change in fear and anxiety was positively associated with changes in meal timing regularity $(\mathrm{r}=0.129, \mathrm{p}=0.019)$ and carbohydrate consumption $(\mathrm{r}=0.127, \mathrm{p}=0.021)$. The change in depressed mood was positively associated with changes in carbohydrate consumption $(\mathrm{r}=0.142, \mathrm{p}=0.010)$ and alcohol $(\mathrm{r}=0.161, \mathrm{p}=0.037)$. In addition, changes in psychological stress were positively associated with changes in consumption of carbohydrates ( $r=$ $0.183, \mathrm{p}=0.001)$, snacks $(\mathrm{r}=0.151, \mathrm{p}=0.008)$, and sedentary time $(\mathrm{r}=0.158, \mathrm{p}=0.004)$.

\section{Discussion}

In this study, we demonstrated considerable lifestyle and mental health changes and the independent impact of the lifestyle changes on glycemic and body weight control during the medium-term period of the COVID-19 pandemic in Japan. In addition, we also demonstrated an association between mental health and lifestyle changes.

Based on the study's findings, we confirmed that dietary habits worsened and activities of daily living decreased in approximately $20 \%$ and $30 \%$ of patients, respectively. In addition, the study demonstrated that irregular meal timing was positively associated with changes in HbA1c levels during the COVID-19 pandemic. Furthermore, decreased walking time was associated with changes in body weight. As is well known, dietary habits and activities of daily living are quite important factors for glycemic and body weight control in patients with diabetes $(5,6)$. Recent studies have revealed that lifestyle changes during the early phase of the COVID-19 pandemic influenced glycemic and body weight control in patients with diabetes in core pandemic areas (7-9). Kishimoto et al. investigated lifestyle changes, including dietary habits and physical activity, body weight, and HbA1c levels in 168 patients with diabetes (7). They revealed that physical activity levels (coded as increased, no change, and decreased) and dietary habits (coded as improved, no change, and deteriorated) were significant determinants of group categorization (patients with elevated $\mathrm{HbA}$ 1c levels $>0.2 \%$ and decreased HbAlc levels $>0.2 \%$ ) in the multiple logistic regression analyses. However, their analysis did not include individual components of dietary habits and physical activities, unlike our report. In addition, changes in body weight have not been analyzed. Munekawa et al. evaluated the association of changes in dietary habits quantitatively, including total diet, snacks, prepared food, and exercise, with changes in HbA1c and body weight in 203 patients with diabetes, using a visual analogue scale (8). They revealed that the total diet intake was positively associated with changes in $\mathrm{HbA} 1 \mathrm{c}$ and that total diet intake and snack consumption were positively associated while exercise was negatively associated with changes in body weight, findings that are partially consistent with those of our study. However, these associations were unadjusted, and whether or not these factors are independently related to $\mathrm{HbA1c}$ and body weight changes is unclear.

In contrast, Takahara et al. evaluated the association of detailed lifestyle changes with HbA1c and body weight changes in 1,402 patients with diabetes using a linear regression model (9). They reported that the change in leisure time for physical activities was inversely associated with HbAlc and weight changes. In contrast, the quantitative change in meals, with a decline in meals eaten out and snacks, was positively associated with HbA1c and weight changes. However, in their reports, physical activity was not analyzed using the quantified indicators used in our study.

These previous studies were performed during the early stage of the COVID-19 pandemic. However, the present study was performed during the medium-term period of about half a year after the COVID-19 pandemic onset, a major difference from previous studies. We observed worsening of dietary habits and a decrease in activities of daily living in a considerable number of patients. Such changes may have led to a deterioration in glycemic control and weight gain, which are generally consistent with previous studies conducted during the early stage of the pandemic. Conversely, the mean $\mathrm{HbA} 1 \mathrm{c}$ during the pandemic was significantly lower than before the pandemic. This improvement may have been due in part to patients' medical guidance from attending physicians and/or their self-care behavior in glycemic control during the pandemic. Thus, considering these findings, including our own, physicians should pay close attention to lifestyle changes leading to worsening glycemic control and body weight gain and provide careful dietary and exercise guidance to diabetic patients while the COVID-19 pandemic persists.

Regarding mental health changes, we confirmed that over $50 \%$ of patients had deteriorated mental health. A recent study reported that as many as $87 \%$ of people with diabetes were affected by psychological stress during the initial stage of the COVID-19 pandemic (11). It is well known that patients with diabetes tend to have more psychological problems, including anxiety, depression, and stress, than the general population (16). In addition, diabetes has been associated with higher severity and mortality rates due to COVID$19(17,18)$. Furthermore, because such warnings were 
Table 4. The Association of Changes in Lifestyles and Mental Health with the Changes in Body Weight $(n=340)$.

\begin{tabular}{|c|c|c|c|c|c|c|}
\hline & \multicolumn{2}{|c|}{ Univariate } & \multicolumn{2}{|c|}{$\begin{array}{l}\text { Regression coefficient } \\
\text { in ordinal variables }\end{array}$} & \multicolumn{2}{|c|}{ Multivariate } \\
\hline & $\mathrm{r}$ & $\mathrm{p}$ & $\beta$ & $\mathrm{p}$ & $\beta$ & $\mathrm{p}$ \\
\hline Age (years) & 0.025 & 0.651 & & & 0.043 & 0.692 \\
\hline $\operatorname{Sex}(0=$ Female, $1=$ Male $)$ & 0.034 & 0.224 & & & 0.092 & 0.316 \\
\hline Duration of diabetes (years) & -0.064 & 0.239 & & & -0.003 & 0.973 \\
\hline $\operatorname{BMI}\left(\mathrm{kg} / \mathrm{m}^{2}\right)$ & 0.187 & 0.001 & & & 0.085 & 0.449 \\
\hline Employment $(0=$ No, $1=$ Yes $)$ & -0.025 & 0.645 & & & & \\
\hline \multicolumn{7}{|l|}{ Change in hypoglycemic agents } \\
\hline Unchanged/modified vs. strengthened & & & 0.034 & 0.533 & -0.087 & 0.387 \\
\hline Attenuated vs. unchanged/modified & & & 0.025 & 0.350 & -0.001 & 0.994 \\
\hline \multicolumn{7}{|l|}{ Changes in dietary habits } \\
\hline \multicolumn{7}{|l|}{ Regularity of meal timing } \\
\hline Unchanged vs. became regular & & & -0.028 & 0.613 & -0.072 & 0.498 \\
\hline Became irregular vs. unchanged & & & 0.190 & 0.001 & 0.110 & 0.274 \\
\hline \multicolumn{7}{|l|}{ Amount of total diet } \\
\hline Unchanged vs. decreased & & & 0.089 & 0.104 & -0.040 & 0.709 \\
\hline Increased vs. unchanged & & & 0.158 & 0.004 & 0.028 & 0.818 \\
\hline \multicolumn{7}{|l|}{ Consumption of carbohydrates } \\
\hline Unchanged vs. decreased & & & 0.064 & 0.247 & 0.184 & 0.104 \\
\hline Increased vs. unchanged & & & 0.120 & 0.029 & 0.053 & 0.626 \\
\hline \multicolumn{7}{|l|}{ Consumption of snacks } \\
\hline Unchanged vs. decreased & & & -0.068 & 0.227 & 0.004 & 0.969 \\
\hline Increased vs. unchanged & & & -0.038 & 0.498 & -0.025 & 0.797 \\
\hline \multicolumn{7}{|l|}{ Consumption of fruits } \\
\hline Unchanged vs. decreased & & & -0.029 & 0.610 & -0.064 & 0.533 \\
\hline Increased vs. unchanged & & & -0.022 & 0.691 & -0.104 & 0.290 \\
\hline \multicolumn{7}{|l|}{ Consumption of alcohol } \\
\hline Unchanged vs. decreased & & & -0.098 & 0.213 & -0.102 & 0.338 \\
\hline Increased vs. unchanged & & & -0.017 & 0.828 & -0.067 & 0.499 \\
\hline \multicolumn{7}{|l|}{ Changes in activities of daily living } \\
\hline Vigorous intensive activity & -0.017 & 0.764 & & & -0.045 & 0.739 \\
\hline Moderate intensive activity & -0.045 & 0.427 & & & 0.068 & 0.605 \\
\hline Walking & -0.154 & 0.008 & & & -0.245 & 0.025 \\
\hline Total activity & -0.062 & 0.297 & & & & \\
\hline Sedentary time & 0.080 & 0.151 & & & -0.045 & 0.677 \\
\hline \multicolumn{7}{|l|}{ Changes in mental health } \\
\hline \multicolumn{7}{|l|}{ Fear and anxiety } \\
\hline Unchanged vs. decreased & & & -0.035 & 0.517 & -0.035 & 0.756 \\
\hline Increased vs. unchanged & & & 0.162 & 0.003 & 0.052 & 0.651 \\
\hline \multicolumn{7}{|l|}{ Depressive mood } \\
\hline Unchanged vs. decreased & & & -0.036 & 0.509 & -0.176 & 0.344 \\
\hline Increased vs. unchanged & & & 0.103 & 0.061 & -0.041 & 0.753 \\
\hline \multicolumn{7}{|l|}{ Psychological stress } \\
\hline Unchanged vs. decreased & & & 0.020 & 0.712 & 0.186 & 0.288 \\
\hline Increased vs. unchanged & & & 0.122 & 0.026 & 0.115 & 0.356 \\
\hline
\end{tabular}

HbA1c: glycated hemoglobin A1c, BMI: body mass index

Change in hypoglycemic agents was coded as "strengthened" (-1 point), "unchanged/modified" (0 point), and "attenuated" (1 point).

Each lifestyle change was coded as "decreased/became regular" (-1 point), "unchanged" (0 point), and "increased/became irregular" (1 point).

widely disseminated through the mass media in Japan, fear of transmission may have deteriorated the mental health of patients with diabetes. Indeed, a recent meta-analysis re- vealed that patients with noninfectious chronic diseases, including diabetes, had a higher risk of depression and anxiety than others during the COVID-19 pandemic (19). 
Table 5. The Correlation between Mental Health and Lifestyle Changes.

\begin{tabular}{|c|c|c|c|c|c|c|c|c|}
\hline & $\begin{array}{c}\text { Regularity of } \\
\text { meal timing }\end{array}$ & $\begin{array}{c}\text { Amount of } \\
\text { total diet }\end{array}$ & $\begin{array}{c}\text { Consumption of } \\
\text { carbohydrates }\end{array}$ & $\begin{array}{l}\text { Consumption } \\
\text { of snacks }\end{array}$ & $\begin{array}{l}\text { Consumption } \\
\text { of fruits }\end{array}$ & $\begin{array}{l}\text { Consumption } \\
\text { of alcohol }\end{array}$ & $\begin{array}{c}\text { Total physical } \\
\text { activity }\end{array}$ & $\begin{array}{l}\text { Sedentary } \\
\text { time }\end{array}$ \\
\hline \multirow{2}{*}{$\begin{array}{l}\text { Fear and } \\
\text { anxiety }\end{array}$} & $\mathrm{r}=0.129$ & $\mathrm{r}=0.036$ & $\mathrm{r}=0.127$ & $\mathrm{r}=-0.098$ & $\mathrm{r}=0.054$ & $\mathrm{r}=0.115$ & $\mathrm{r}=-0.051$ & $\mathrm{r}=0.103$ \\
\hline & $\mathrm{p}=0.019$ & $\mathrm{p}=0.514$ & $\mathrm{p}=0.021$ & $\mathrm{p}=0.077$ & $\mathrm{p}=0.332$ & $\mathrm{p}=0.138$ & $\mathrm{p}=0.391$ & $\mathrm{p}=0.064$ \\
\hline \multirow{2}{*}{$\begin{array}{l}\text { Depressed } \\
\text { mood }\end{array}$} & $\mathrm{r}=-0.004$ & $\mathrm{r}=0.106$ & $\mathrm{r}=0.142$ & $\mathrm{r}=-0.086$ & $\mathrm{r}=0.086$ & $\mathrm{r}=0.161$ & $\mathrm{r}=-0.058$ & $\mathrm{r}=0.106$ \\
\hline & $\mathrm{p}=0.943$ & $\mathrm{p}=0.055$ & $\mathrm{p}=0.010$ & $\mathrm{p}=0.118$ & $\mathrm{p}=0.118$ & $\mathrm{p}=0.037$ & $\mathrm{p}=0.331$ & $\mathrm{p}=0.057$ \\
\hline \multirow{2}{*}{$\begin{array}{l}\text { Psychological } \\
\text { stress }\end{array}$} & $\mathrm{r}=0.078$ & $\mathrm{r}=0.080$ & $\mathrm{r}=0.183$ & $\mathrm{r}=0.151$ & $\mathrm{r}=0.038$ & $\mathrm{r}=0.160$ & $\mathrm{r}=-0.024$ & $\mathrm{r}=0.158$ \\
\hline & $\mathrm{p}=0.156$ & $\mathrm{p}=0.148$ & $\mathrm{p}=0.001$ & $\mathrm{p}=0.008$ & $\mathrm{p}=0.492$ & $\mathrm{p}=0.172$ & $\mathrm{p}=0.682$ & $\mathrm{p}=0.004$ \\
\hline
\end{tabular}

HbA1c: glycated hemoglobin A1c

Although there was no significant association between changes in mental health and glycemic control in this study, mental health deterioration was associated with worsening of dietary habits, such as increased carbohydrates and snacks. Recent studies have reported an association between changes in mental health and dietary habits in patients with diabetes during the COVID-19 pandemic $(8,12)$. Sanker et al. reported that $15.5 \%$ of patients had increased mental stress during the lockdown. The majority had an unhealthy dietary pattern, including higher consumption of snacks, which was consistent with our study findings (12). Munekawa et al. also reported that $41.9 \%$ of patients experienced increased stress during the early phase of the COVID-19 pandemic, and this was positively associated with changes in prepared food intake (8). The causal relationship between mental health deterioration and increased consumption of carbohydrates and snacks may be attributed to the finding that negative emotion is likely to cause emotional hunger and eating. In addition, carbohydrate ingestion stimulates serotonin production, which enhances mood and alleviates stress (20, 21).

Regarding the association between mental health and activities of daily living, we found an association between increased psychological stress and sedentary time. Stanton et al. reported that higher depression, anxiety, and stress symptoms were associated with decreased physical activity during the COVID-19 pandemic, based on a study of 1,491 Australian adults (22). Although the association between mental health and activities of daily living is bidirectional (23), deterioration of mental health may be more likely to lead to a decrease in activities of daily living during the pandemic.

Given these findings, the deterioration of mental health may lead indirectly to worsened glycemic control through the worsening of self-care, including dietary habits and activities of daily living during the pandemic. Unfortunately, we found no significant association between changes in mental health and glycemic control in this study; however, negative psychological factors are directly associated with the worsening of glycemic control through increased generation of stress hormones, including cortisol $(24,25)$. However, a systematic review and meta-analysis showed that psychosocial interventions modestly but significantly improved glycemic control and mental health outcomes (26). Therefore, we believe that physicians should pay close attention to changes in mental health and lifestyle changes while the COVID-19 pandemic persists.

Several limitations associated with the present study warrant mention. First, the responses to the questions were dependent on memory and the patients' subjective opinions, which may have affected the outcome validity. Second, this study was performed in a single diabetes center in Japan, with a relatively small sample size and no control. Third, we used the IPAQ-SF, a self-report questionnaire that assesses physical activity and has been accurately validated in people 15-69 years old. However, our study population included elderly people over 70 years old. This data collection tool has only recently been used in clinical practice to evaluate this age group $(27,28)$. Fourth, the COVID-19 infection rate was lower and measures employed in the study area less stringent than those in Western countries and other regions with government-enforced lockdowns and quarantines. Therefore, these results may not be representative of other populations. Furthermore, this study did not include patients whose visits to our department had been suspended due to the pandemic. Those patients may have been experiencing more lifestyle changes related to dietary habits, activities of daily living, and mental health. Therefore, the present results may have been underestimated. Finally, although we detected an association between lifestyle changes and glycemic and body weight control, and changes in mental health and dietary habits, we were unable to clarify any causal relationship because of the cross-sectional design of this study. Therefore, further prospective, large-scale clinical studies are needed to clarify the causality. However, we believe that this study's findings will provide important information on diabetes care during the COVID-19 pandemic.

In conclusion, the persistence of the COVID-19 pandemic has had a considerable impact on the lifestyles and mental health of patients with diabetes. In addition, lifestyle changes were shown to be associated with glycemic and body weight control, and mental health changes were associated with lifestyle changes. Thus, physicians need to provide more careful diet and exercise guidance and mental healthcare to patients with diabetes during the COVID-19 pandemic.

\section{Author's disclosure of potential Conflicts of Interest (COI).}

Tetsuyuki Yasuda: Honoraria, Takeda Pharmaceutical, Novartis Pharmaceuticals and Nippon Boehringer Ingelheim. 


\section{Acknowledgement}

We thank Reiko Ohara, Setsuko Nishio, Ryoko Sato, and Saki Hashio of the Division of Nutrition Management, Osaka Police Hospital, for their cooperation in writing this paper.

\section{References}

1. Nussbaumer-Streit B, Mayr V, Dobrescu AI, et al. Quarantine alone or in combination with other public health measures to control COVID-19: a rapid review. Cochrane Database Syst Rev 4: CD013574, 2020.

2. Japanese Ministry of Health Labour and Welfare. About coronavirus disease 2019 (COVID-19) [Internet]. 2020 [cited 2020 Dec 31]. Available from: https://www.mhlw.go.jp/stf/covid-19/open-dat a.html (in Japanese)

3. Suzuki Y, Maeda N, Hirado D, Shirakawa T, Urabe Y. Physical activity changes and its risk factors among community dwelling Japanese older adults during the COVID-19 epidemic: associations with subjective well-being and health-related quality of life. Int $\mathrm{J}$ Environ Res Public Health 17: 6951, 2020.

4. Awano N, Oyama N, Akiyama K, et al. Anxiety, depression, and resilience of healthcare workers in Japan during the coronavirus disease 2019 outbreak. Intern Med 59: 2693-2699, 2020.

5. Magkos F, Yannakoulia M, Chan JL, et al. Management of the metabolic syndrome and type 2 diabetes through lifestyle modification. Annu Rev Nutr 29: 223-256, 2009.

6. American Diabetes Association. Facilitating behavior change and well-being to improve health outcomes: Standards of Medical Care in Diabetes-2021. Diabetes Care 44 (Supplement 1): S53-S72, 2021.

7. Kishimoto M, Ishikawa T, Odawara M. Behavioral changes in patients with diabetes during the COVID-19 pandemic. Diabetol Int 12: 1-5, 2020.

8. Munekawa C, Hosomi Y, Hashimoto Y, et al. Effect of coronavirus disease 2019 pandemic on the lifestyle and glycemic control in patients with type 2 diabetes: a cross-section and retrospective cohort study. Endocr J 68: 201-210, 2021.

9. Takahara M, Watanabe H, Shiraiwa T, et al. Lifestyle changes and their impact on glycemic control and weight control in patients with diabetes during the coronavirus disease 2019 pandemic in Japan. J Diabetes Investig 2021. Forthcoming.

10. Khare J, Jindal S. Observational study on effect of lock down due to COVID 19 on glycemic control in patients with diabetes: experience from central India. Diabetes Metab Syndr 14: 1571-1574, 2020.

11. Ghosh A, Arora B, Gupta R, Anoop S, Misra A. Effects of nationwide lockdown during COVID-19 epidemic on lifestyle and other medical issues of patients with type 2 diabetes in north India. Diabetes Metab Syndr 14: 917-920, 2020.

12. Sankar P, Ahmed WN, Mariam Koshy V, Jacob R, Sasidharan S. Effects of COVID-19 lockdown on type 2 diabetes, lifestyle and psychosocial health: a hospital-based cross-sectional survey from South India. Diabetes Metab Syndr 14: 1815-1819, 2020.

13. Grabia M, Markiewicz-Żukowska R, Puścion-Jakubik A, et al. The nutritional and health effects of the COVID-19 pandemic on patients with diabetes mellitus. Nutrients 12: 3013, 2020.

14. Ruiz-Roso MB, Knott-Torcal C, Matilla-Escalante DC, et al.
COVID-19 lockdown and changes of the dietary pattern and physical activity habits in a cohort of patients with type 2 diabetes mellitus. Nutrients 12: 2327, 2020.

15. Craig CL, Marshall AL, Sjöström M, et al. International physical activity questionnaire: 12-country reliability and validity. Med Sci Sports Exerc 35: 1381-1395, 2003.

16. Fisher L, Hessler D, Polonsky W, Strycker L, Masharani U, Peters A. Diabetes distress in adults with type 1 diabetes: prevalence, incidence and change over time. J Diabetes Complications 30: 11231128, 2016.

17. Zhang Y, Cui Y, Shen M, et al. Association of diabetes mellitus with disease severity and prognosis in COVID-19: a retrospective cohort study. Diabetes Res Clin Pract 165: 108227, 2020.

18. Zhu L, She ZG, Cheng X, et al. Association of blood glucose control and outcomes in patients with COVID-19 and pre-existing type 2 diabetes. Cell Metab 31: 1068-1077, 2020.

19. Wu T, Jia X, Shi H, et al. Prevalence of mental health problems during the COVID-19 pandemic: a systematic review and metaanalysis. J Affect Disord 281: 91-98, 2021.

20. Litwin R, Goldbacher EC, Cardaciotto L, Gambrel LE. Negative emotions and emotional eating: the mediating role of experiential avoidance. Eat Weight Disord 22: 97-104, 2016.

21. Wurtman JJ. The involvement of brain serotonin in excessive carbohydrate snacking by obese carbohydrate cravers. J Am Diet Assoc 84: 1004-1007, 1984.

22. Stanton R, To QG, Khalesi S, et al. Depression, anxiety and stress during COVID-19: associations with changes in physical activity, sleep, tobacco and alcohol use in Australian adults. Int $\mathrm{J}$ Environ Res Public Health 17: 4065, 2020.

23. Bernard P, Doré I, Romain AJ, Hains-Monfette G, Kingsbury C, Sabiston C. Dose response association of objective physical activity with mental health in a representative national sample of adults: a cross-sectional study. PLoS One 13: e0204682, 2018.

24. Naranjo D, Hessler DM, Deol R, Chesla CA. Health and psychosocial outcomes in U.S. adult patients with diabetes from diverse ethnicities. Curr Diab Rep 12: 729-738, 2012.

25. Weber B, Schweiger U, Deuschle M, Heuser I. Major depression and impaired glucose tolerance. Exp Clin Endocrin Diabetes 108: 187-190, 2000.

26. Harkness E, Macdonald W, Valderas J, Coventry P, Gask L, Bower P. Identifying psychosocial interventions that improve both physical and mental health in patients with diabetes: a systematic review and meta-analysis. Diabetes Care 33: 926-930, 2010.

27. Joseph KL, Dagfinrud H, Christie A, Hagen KB, Tveter AT. Criterion validity of The International Physical Activity QuestionnaireShort Form (IPAQ-SF) for use in clinical practice in patients with osteoarthritis. BMC Musculoskelet Disord 22: 232, 2021.

28. Mori H, Kuroda A, Yoshida S, et al. High prevalence and clinical impact of dynapenia and sarcopenia in Japanese patients with type 1 and type 2 diabetes: Findings from the Impact of Diabetes Mellitus on Dynapenia study. J Diabetes Investig 12: 1050-1059, 2021.

The Internal Medicine is an Open Access journal distributed under the Creative Commons Attribution-NonCommercial-NoDerivatives 4.0 International License. To view the details of this license, please visit (https://creativecommons.org/licenses/ by-nc-nd/4.0/).

\section{(C) 2022 The Japanese Society of Internal Medicine} Intern Med 61: 303-311, 2022 